\title{
Association of Multidrug Resistance in Epilepsy with a Polymorphism in the Drug-Transporter Gene ABCB1
}

\author{
Asra Siddiqui, M.R.C.P., Reinhold Kerb, Ph.D., Michael E. Weale, Ph.D., \\ Ulrich Brinkmann, Ph.D., Alice Smith, B.Sc., David B. Goldstein, Ph.D., \\ Nicholas W. Wood, F.R.C.P., Ph.D., and Sanjay M. Sisodiya, M.R.C.P., Ph.D.
}

\section{ABSTRACT}

From the Departments of Molecular Pathogenesis (A. Siddiqui, N.W.W.) and Clinical and Experimental Epilepsy (A. Siddiqui, S.M.S.), Institute of Neurology, and the Department of Biology (A. Smith, D.B.G.), University College London, London; Genostics, London (M.E.W.); the Pharmacogenetics Laboratory, Epidauros Biotechnology, Bernried, Germany (R.K., U.B.); and the Nationa Neurological Institute, Singapore (M.E.W. D.B.G., N.W.W.). Address reprint requests to Dr. Sisodiya at the Department of Clinical and Experimental Epilepsy, UCL Institute of Neurology, London WCIN 3BG, United Kingdom, or at s.sisodiya@ion.ucl.ac.uk.

N Engl J Med 2003;348:1442-8.

Copyright ( 2003 Massachusetts Medical Society.

\section{BACK GROU N D}

One third of patients with epilepsy have drug-resistant epilepsy, which is associated with an increased risk of death and debilitating psychosocial consequences. Because this form is resistant to multiple antiepileptic drugs, the mode of resistance must be nonspecific, involving drug-efflux transporters such as ATP-binding cassette sub-family B member 1 (ABCB1, also known as MDR1 and P-glycoprotein 170). We hypothesized that the CC genotype at the ABCB1 C3435T polymorphism, which is associated with increased expression of the protein, influences the response to antiepileptic-drug treatment.

\section{METHODS}

ABCB1 3435 was genotyped in 315 patients with epilepsy, classified as drug-resistant in 200 and drug-responsive in 115, and 200 control subjects without epilepsy. Recently devised methods were used to control for population stratification, and linkage disequilibrium was calculated across the gene.

\section{RESULTS}

As compared with patients with drug-responsive epilepsy, patients with drug-resistant epilepsy were more likely to have the CC genotype at ABCB1 3435 than the TT genotype (odds ratio, 2.66; 95 percent confidence interval, 1.32 to $5.38 ; \mathrm{P}=0.006$ ). There was no genetic stratification between the two groups of patients. The polymorphism fell within an extensive block of linkage disequilibrium spanning much or all of the gene, implying that the polymorphism may not itself be causal but rather may be linked with the causal variant.

CONCLUSIONS

These pharmacogenomic results identify a genetic factor associated with resistance to antiepileptic drugs. 
T HE CONTINUED OCCURRENCE OF SEIzures despite the use of several antiepileptic drugs, even as polytherapy at maximal tolerated doses, is a major health problem and increases the risk of death from epilepsy. None of the reported associations with clinical drug resistance, such as remote symptomatic causes, early onset, multiple seizure types, and a high frequency of seizures before the initiation of treatment, provide a mechanistic explanation of the phenomenon. ${ }^{1-3}$ In addition, it is not clear why the same type of epilepsy may be drug-resistant in one person and drugresponsive in another and whether this is a pharmacogenomic phenomenon.

Drug resistance in epilepsy constitutes a true multidrug-resistance phenotype, since antiepileptic drugs act through diverse mechanisms. Most antiepileptic drugs are planar lipophilic agents and thus theoretical substrates for the ATP-binding cassette subfamily B member 1 (ABCB1) transporter (also known as MDR1 and P-glycoprotein 170), the upregulation of which causes a multidrug-resistance phenotype in cancer cells. ${ }^{4} \mathrm{ABCB} 1$ can transport at least four antiepileptic drugs. ${ }^{5}$ In patients with epilepsy, the multidrug-resistance phenotype is associated with increased lesional $A B C B 1$ levels in resected brain tissue. ${ }^{6-11}$ Thus, the $A B C B 1$ gene is an important candidate gene potentially influencing the response to antiepileptic drugs. To evaluate its involvement in drug resistance in epilepsy and explore its pharmacogenomics, we typed the $A B C B 1$ polymorphism ( $C$ to $\mathrm{T}$ ) at position 3435 in $315 \mathrm{pa}-$ tients with epilepsy classified as either drug-resistant or drug-responsive. The CC genotype is associated with both increased levels and increased activity of $A B C B 1$ protein. ${ }^{12}$

\section{METHODS}

\section{SUBJECTS}

The study was approved by the joint research ethics committee of the National Hospital for Neurology and Neurosurgery and the University College London Institute of Neurology in London. All patients gave written informed consent. All patients were recruited through the same epilepsy clinic at the National Hospital for Neurology and Neurosurgery and were consecutive patients with an established clinical diagnosis of epilepsy (defined by the occurrence of two or more unprovoked seizures). Patients were eligible if they had either drug-resistant or drug-responsive epilepsy according to the following definitions and criteria. Drug resistance was defined as the occurrence of at least four seizures over the year before recruitment with trials of more than three appropriate antiepileptic drugs at maximal tolerated doses, which were established on the basis of the occurrence of clinical side effects at supramaximal doses. Where it was possible, only the recognized, more specific drugs for a syndrome were considered appropriate - for example, failure of a case of primary generalized epilepsy to respond to carbamazepine was not considered a failure for the purposes of this study.

Drug responsiveness was defined as complete freedom from seizures for at least a year, up to the date of the last follow-up visit, in patients with epilepsy treated with antiepileptic drugs. Of the patients who were invited to participate, six declined (less than 2 percent). Control subjects were 200 consecutive white patients with neurologic conditions without epilepsy who were identified from a DNA repository at the Institute of Neurology. Patients who had undergone surgical treatment for their drug-resistant epilepsy were classified as having drug-resistant disease irrespective of the outcome of surgery. All DNA was analyzed and all studies were conducted in a blinded fashion.

\section{GENOTYPING AT ABCB1 3435}

DNA was extracted from blood samples according to standard methods. Samples from the first 262 patients and 90 controls were genotyped by Epidauros by an automated method on purified genomic DNA by a TaqMan assay specifically devised to distinguish the C3435T polymorphism of the human ABCB1 gene. The C3435T polymorphism was analyzed in samples from the remaining patients and controls in the United Kingdom by direct sequencing with use of information supplied by Epidauros. Amplified polymerase-chain-reaction (PCR) products were sequenced with the use of forward and reverse primers, the Big Dye Terminator cycle sequencing kit (Perkin Elmer), and an automated sequencer (model 377, ABI). The sequences were analyzed with use of the Sequence Navigator program (Perkin Elmer).

\section{GENOMIC CONTROL}

To address the possibility that any observed differences in allele frequency between drug-resistant and drug-responsive populations might be due to an unsuspected population structure, we used a set of genetic markers with intermediate allele frequencies 
that were not linked to ABCB1. The markers used polymorphic Alu insertion markers - code for variable lengths of amino acid residue repeats.

Nine polymorphic Alu insertion markers were typed on the basis of their heterozygosity and location in the human genome. ${ }^{13,14}$ Alu insertion polymorphisms were amplified by PCR in all 315 patients with epilepsy. In a 50- $\mu \mathrm{l}$ reaction, 50 to $100 \mathrm{ng}$ of genomic DNA was used with 40 pmol of each primer, $2.5 \mathrm{mM}$ of deoxynucleoside triphosphate, Promega Taq DNA polymerase $(0.2 \mathrm{U})$, and $5 \mu \mathrm{l}$ of Taq DNA polymerase buffer (containing $1.2 \mathrm{mM}$ magnesium chloride). Samples were amplified with the following cycles: initial denaturation of $4 \mathrm{~min}$ utes at $95^{\circ} \mathrm{C}$, then 1 minute of denaturation $\left(95^{\circ} \mathrm{C}\right)$, 1 minute at the annealing temperature, and $1 \mathrm{~min}$ ute of extension $\left(72^{\circ} \mathrm{C}\right)$, repeated for 35 cycles and followed by another 10-minute period of extension $\left(72^{\circ} \mathrm{C}\right)$. PCR products were run on agarose gels with ethidium bromide and analyzed under ultraviolet light. Two Alu markers showed significant deviation from the expected population distribution (HardyWeinberg equilibrium), owing to genotyping errors. Results from these markers were therefore not included in the analysis: their inclusion, however, did not change the overall results.

\section{CALCULATION OF LINKAGE DISEQUILIBRIUM}

Resequencing was carried out on 32 Centre d'Etude du Polymorphisme Humain trios (mother, father, and child) to genotype single-nucleotide polymorphisms in intron 2 (single-nucleotide polymorphism identity rs2888599; available at http://www. ncbi.nlm.nih.gov/SNP/), exon 12 (C1236T and

\begin{tabular}{|c|c|c|c|c|}
\hline \multirow[t]{2}{*}{ Phenotype } & \multirow[t]{2}{*}{$\begin{array}{l}\text { Total } \\
\text { No. }\end{array}$} & \multicolumn{3}{|c|}{ ABCB1 3435 Genotype } \\
\hline & & $\mathrm{CC}$ & $\begin{array}{c}\mathrm{CT} \\
\text { no. (\%) }\end{array}$ & TT \\
\hline Drug-resistant epilepsy & 200 & $55(27.5)$ & $106(53.0)$ & $39(19.5)$ \\
\hline Drug-responsive epilepsy & 115 & $18(15.7)$ & $63(54.8)$ & $34(29.6)$ \\
\hline Control & 200 & $37(18.5)$ & $116(58.0)$ & $47(23.5)$ \\
\hline
\end{tabular}

* For all 315 patients with epilepsy, patients with drug-resistant epilepsy were more likely than those with drug-responsive epilepsy to have the CC genotype than the TT genotype $\left(\chi^{2}=7.65, P=0.006\right)$. The results were similar when the analysis was restricted to the 297 white patients $\left(\chi^{2}=6.66 ; \mathrm{P}=0.01\right.$; odds ratio, 2.58; 95 percent confidence interval, 1.25 to 5.36 ).
$\mathrm{C}+44 \mathrm{~T}),{ }^{12,15}$ exon 21 (G2677A), ${ }^{16}$ and exon 26 (C3435T). ${ }^{12}$ PCR conditions were as follows: 15 minutes of initial denaturation at $95^{\circ} \mathrm{C}$, then $35 \mathrm{cy}-$ cles of denaturation $\left(94^{\circ} \mathrm{C}\right)$, annealing $\left(57^{\circ} \mathrm{C}\right)$, and extension $\left(72^{\circ} \mathrm{C}\right)$, each for 30 seconds, and a final 10 -minute period of extension $\left(72^{\circ} \mathrm{C}\right)$. PCR was carried out with 50 ng of DNA, $2.5 \mathrm{mM}$ magnesium chloride, $0.2 \mathrm{mM}$ of each deoxynucleoside triphosphate, $0.5 \mu \mathrm{M}$ of each primer, and $0.25 \mathrm{U}$ of HotStarTaq polymerase (Qiagen), in a total volume of $10 \mu \mathrm{l}$. PCR products were sequenced in the forward direction with the Big Dye Terminator cycle sequencing kit, cleaned by standard isopropanol precipitation and analyzed with an ABI Prism automated sequencer (model 3700, Applied Biosystems).

\section{STATISTICAL ANALYSIS}

Allele frequencies were compared between populations with use of the chi-square test. Observed and expected allele frequencies within populations were compared by means of the Hardy-Weinberg test. Correction for genetic stratification was undertaken with use of the method of Reich and Goldstein. ${ }^{17}$ All reported P values are two-sided; a P value of less than 0.05 was considered to indicate statistical significance.

\section{RESULTS}

As compared with the 115 patients with drugresponsive epilepsy, the 200 patients with drugresistant epilepsy were more likely to have the CC than the TT genotype (odds ratio, 2.66; 95 percent confidence interval, 1.32 to $5.38 ; \mathrm{P}=0.006$ ) (Table 1). The frequency of the CT genotype did not differ significantly between the two groups. Thus, the $\mathrm{C}$ polymorphism was overrepresented among patients with drug-resistant epilepsy as compared with those with drug-responsive epilepsy ( $\mathrm{P}=0.008)$. As compared with the 200 control subjects without epilepsy, patients with drug-resistant epilepsy had a higher frequency of the CC genotype and a lower frequency of the TT genotype $(\mathrm{P}=0.05)$. The frequencies in the drug-responsive group did not differ significantly from those of ethnically matched control subjects or published reports. ${ }^{15}$ No significant deviations from Hardy-Weinberg equilibrium were observed in either group of patients $(\mathrm{P}=0.39$ for the drug-resistant group and $\mathrm{P}=0.26$ for the drug-responsive group).

Unexpected differences in genetic backgrounds 
between study groups - or population stratification - may result in spurious associations. To minimize overt population stratification, all subjects were from the same hospital clinic. In the drugresistant and drug-responsive groups, 187 and 110 patients were white, respectively, and 13 and 5 patients were Asian, respectively $(\mathrm{P}=0.43)$. The association persisted when the analysis was confined to white patients (Table 1). There was no significant difference between those with drug-resistant epilepsy and those with drug-responsive epilepsy with respect to proportions of partial, generalized, or unclassified epilepsy syndromes (data not shown).

To determine whether drug-resistant and drugresponsive groups might be covertly stratified, we analyzed unlinked genomic-DNA markers (Alu insertion markers) in disparate positions across the genome. There were no significant deviations from Hardy-Weinberg equilibrium for the seven markers within either the drug-resistant or drug-responsive group (Table 2). The mean $\chi^{2}$ statistic for the comparison of allele frequencies between the drugresistant and drug-responsive groups was 0.94 , implying that the two cohorts were not significantly stratified. As an additional correction for stratification, ${ }^{17}$ we divided the observed $\chi^{2}$ by the maximal $\chi^{2}$ across unlinked sites ${ }^{17}$ consistent with the observed mean value of 0.96 and found that the $A B C B 1$ $3435 \mathrm{C}$ allele remained significantly overrepresented among patients with drug-resistant epilepsy as compared with those with drug-responsive epilepsy $(\mathrm{P}<0.05)$.

Genotyping in 32 European trios (mother, father, and child) of $A B C B 1$ polymorphisms in intron 2 and exons 12a, 12b, 21, and 26 showed a high degree of linkage disequilibrium spanning at least between 12a and 26 (Table 3). The linkage disequilibrium may also extend upstream of exon 12 , but the marker upstream of exon 12 was not sufficiently polymorphic to estimate the level of association. A simple pattern of groupings of single-nucleotide polymorphisms, or haplotype, was observed, in which five common haplotypes accounted for 88.5 percent of parental chromosomes for which complete phase information was available (haplotypes A, B, C, D, and E) (Table 4).

\section{I SCUSSION}

A role for $\mathrm{ABCB} 1$ in resistance to treatment of epilepsy has biologic plausibility. ABCB1 contributes to drug resistance in other diseases, including many
Table 2. Distribution of Alu Insertion Polymorphisms among the Patients with Drug-Resistant and Drug-Responsive Epilepsy.

\begin{tabular}{|c|c|c|c|c|c|}
\hline $\begin{array}{l}\text { Alu Insertion } \\
\text { Polymorphism } \\
\text { and Group }\end{array}$ & $\begin{array}{c}\text { Chromosome } \\
\text { No. }\end{array}$ & $\stackrel{+/+}{\text { Genotype }}$ & $\begin{array}{c}+/- \\
\text { Genotype } \\
\text { 10. of patients }\end{array}$ & $\begin{array}{l}-/- \\
\text { Genotype }\end{array}$ & $\begin{array}{c}\mathbf{P} \\
\text { Value*: }\end{array}$ \\
\hline YCIRG68 & 7 & & & & 0.17 \\
\hline Drug-resistant & & 15 & 56 & 129 & \\
\hline Drug-responsive & & 6 & 27 & 82 & \\
\hline YB8NBC65 & 6 & & & & 0.57 \\
\hline Drug-resistant & & 21 & 78 & 101 & \\
\hline Drug-responsive & & 13 & 48 & 54 & \\
\hline YA5NBC102 & 17 & & & & 0.16 \\
\hline Drug-resistant & & 14 & 76 & 110 & \\
\hline Drug-responsive & & 5 & 37 & 73 & \\
\hline YA5NBC27 & 11 & & & & 0.19 \\
\hline Drug-resistant & & 20 & 64 & 116 & \\
\hline Drug-responsive & & 8 & 33 & 74 & \\
\hline YA5NBC51 & 3 & & & & 0.88 \\
\hline Drug-resistant & & 41 & 86 & 73 & \\
\hline Drug-responsive & & 26 & 46 & 43 & \\
\hline YA5NBC5 & 2 & & & & 0.90 \\
\hline Drug-resistant & & 12 & 57 & 129 & \\
\hline Drug-responsive & & 7 & 34 & 74 & \\
\hline YA5NBC109 & 22 & & & & 0.73 \\
\hline Drug-resistant & & 40 & 110 & 50 & \\
\hline Drug-responsive & & 23 & 60 & 32 & \\
\hline
\end{tabular}

* The $P$ values relate to the results of $\chi^{2}$ tests with one degree of freedom for differences in allele frequencies between patients with drug-resistant epilepsy and those with drug-responsive epilepsy. A P value of less than 0.05 corresponds to a $\chi^{2}$ value of more than 3.84 .

$\uparrow$ Two patients could not be genotyped.

forms of cancer, ${ }^{19}$ rheumatoid arthritis, ${ }^{20}$ and inflammatory bowel disease. ${ }^{21}$ The postulated role of a gene product in disease pathophysiology may be supported by the demonstration that polymorphisms in the encoding gene can influence that role. In whites, the ABCB1 3435 TT genotype is associated with low levels of ABCB1 in enterocytes, ${ }^{12}$ CD56+ natural killer cells, ${ }^{22}$ and peripheral-blood mononuclear cells. ${ }^{23}$ In comparison to the CC genotype, the TT genotype is also associated with functional restriction, being associated with higher plas- 


\begin{tabular}{|lccccc|}
\hline \hline $\begin{array}{l}\text { Table 3. Pairwise Comparisons for Linkage Disequilibrium } \\
\text { among Five Single-Nucleotide-Polymorphism Loci in ABCB1.* }\end{array}$ & \\
\hline $\begin{array}{l}\text { Locus of Single- } \\
\text { Nucleotide } \\
\text { Polymorphism }\end{array}$ & \multicolumn{5}{c|}{ Locus of Single-Nucleotide Polymorphism } \\
& Intron 2 & Exon 12a & Exon 12b & Exon 21 & Exon 26 \\
Intron 2 & - & $P=1.00$ & $P=1.00$ & $P=1.00$ & $P=0.39$ \\
Exon 12a & $r^{2}=0.01$ & - & $P=0.29$ & $P<0.001$ & $P<0.001$ \\
Exon 12b & $r^{2}=0.002$ & $r^{2}=0.07$ & - & $P=0.06$ & $P=0.006$ \\
Exon 21 & $r^{2}=0.01$ & $r^{2}=0.61$ & $r^{2}=0.06$ & - & $P<0.001$ \\
Exon 26 & $r^{2}=0.02$ & $r^{2}=0.20$ & $r^{2}=0.12$ & $r^{2}=0.40$ & - \\
\hline
\end{tabular}

* The $r^{2}$ values were obtained according to the method of Weir. ${ }^{18}$ The P values were obtained with Fisher's exact test. Genotyping was carried out on 32 white European trios (mother, father, and child).

Table 4. Haplotypes of Five Single-Nucleotide-Polymorphism Loci in ABCB1.

Haplotype
Identification Code

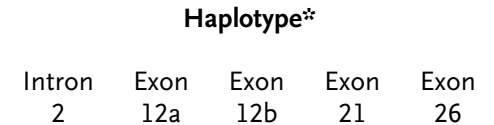

$\begin{array}{ccccccc}\text { A } & 1 & 0 & 1 & 0 & 1 & \% \\ \text { B } & 1 & 1 & 1 & 1 & 0 & 23.6 \\ \text { C } & 1 & 1 & 1 & 1 & 1 & 15.4 \\ \text { D } & 1 & 1 & 1 & 0 & 1 & 7.7 \\ \text { E } & 1 & 1 & 0 & 1 & 0 & 7.7 \\ \text { F } & 1 & 0 & 1 & 1 & 0 & 5.8 \\ \text { G } & 1 & 0 & 1 & 0 & 0 & 3.8 \\ \text { H } & 0 & 1 & 1 & 1 & 0 & 1.9\end{array}$

* The number 1 denotes a major allele, and 0 a minor allele.

$\dagger$ The frequency was calculated with the use of 52 parental haplotypes from 32 white European trios (mother, father, and child) for which complete phase information was available.

ma levels of the substrate digoxin. ${ }^{12}$ Results from other studies are conflicting: one suggested that the TT genotype is associated with increased ABCB1 levels and decreased plasma levels of the substrate fexofenadine in white American and black American subjects, ${ }^{24}$ whereas no difference in plasma fexofenadine levels was seen among white German subjects. ${ }^{25}$ We have demonstrated that the C $3435 \mathrm{~T}$ polymorphism in $A B C B 1$ is associated with drug resistance in epilepsy, irrespective of the type of epilepsy or antiepileptic drug used.

Although the association with variation in the $A B C B 1$ gene is strong, recent evidence that the human genome is structured in blocks of linkage disequilibrium ${ }^{26}$ and the fact that this well-known C3435T polymorphism is silent raise the possibility that the polymorphism is not itself causal but rather in linkage disequilibrium with the causal variant. Our data show evidence of linkage disequilibrium extending from at least exon 26 to exon 12 , and possibly farther. Consistent with other recent evidence, we also showed that the studied region of the ABCB1 gene has a simple haplotype structure, involving five common haplotypes. These common haplotypes formed a consistent network compatible with a process of mutation without recombination (although the rare haplotypes displayed inconsistencies that could have arisen from rare recombination events). Thus, the low $\mathrm{r}^{2}$ values between some single-nucleotide polymorphisms reported in Table 3 do not indicate increased levels of recombination but rather different mutational clades, or groups. This implies that there could be many polymorphisms included within this block of linkage disequilibrium that have high $\mathrm{r}^{2}$ with the $\mathrm{C} 3435 \mathrm{~T}$ polymorphism, if they occurred in a similar part of the genealogy as the C3435T polymorphism. Thus, it may be possible to obtain a reliable clinical prediction of drug resistance without identifying the causal variant, but the possibility that there may be different patterns of association in different populations means that care may be necessary in applying such predictors to heterogeneous populations.

In fact, the extensive linkage disequilibrium across $A B C B 1$ illustrates an analytic disadvantage of the block structure of linkage disequilibrium in the human genome ${ }^{26}$ : association data may not provide precise information about the location of the causal variant. ${ }^{27}$ The final identification of the causal site may require detailed functional analyses. We note, however, that $A B C B 1$ may be unusual in this regard, since it has an intermediate phenotype of high prevalence that could help pinpoint the causal variant. For example, although there is a high level of linkage disequilibrium across the gene, some of the rare haplotypes involving C3435T appear to have been generated by recombination. Thus, if association is incomplete, assessment of ABCB1 levels in persons with rare recombinant haplotypes may help pinpoint the variant responsible for altered expres- 
sion. Such an assessment could be undertaken in resected brain tissue from patients with epilepsy.

There is mechanistic plausibility for the observed association between $\mathrm{ABCB} 1$ and drug-resistant epilepsy. The fluorescence of rhodamine allows quantitative determination of alterations in its intracellular retention caused by changes in the activity of $\mathrm{ABCB} 1$, for which it is a substrate. By comparing the intracellular retention of rhodamine under various controlled conditions, Hitzl et al. determined the influence of the $\mathrm{C} 3435 \mathrm{~T}$ polymorphism on $\mathrm{ABCB} 1$ transport. ${ }^{22}$ In peripheral-blood CD56+ leukocytes from volunteers, they demonstrated reduced rhodamine retention, a marker of increased $\mathrm{ABCB} 1$ drug transport, in leukocytes from subjects with the CC genotype, as compared with subjects with the TT genotype. Leukocytes from subjects with the CT genotype had intermediate function, providing evidence of the existence of a nucleotide-sequencedependent level of $A B C B 1$ function in these subjects. These findings were subsequently confirmed. ${ }^{24}$ Leukocyte levels of ABCB1 messenger RNA showed a similar trend; the highest levels were in subjects with the CC genotype and the lowest levels in subjects with the TT genotype. Rhodamine transport is a robust marker of the drug-transport capacity of ABCB1. ${ }^{28}$ These findings show that the C $3435 \mathrm{~T}$ polymorphism is truly linked to the drug-transport capacity of $\mathrm{ABCB} 1$ and provide an underlying mechanism.

The site of action of the observed association is likely to be within brain parenchyma. ABCB1 is present in the blood-brain barrier, and $A B C B 1$ knockout mice have higher brain levels of $A B C B 1$ substrates than do wild-type mice. ${ }^{29}$ Although the $\mathrm{CC}$ genotype was weakly associated with reduced serum levels of phenytoin, an ABCB1-substrate antiepileptic drug, in Turkish subjects, ${ }^{30}$ all our patients with drug-resistant epilepsy had received their own individual maximal tolerated doses of antiepileptic drugs. Thus, it would seem unlikely that polymorphisms in genes encoding drug-metabolizing proteins could explain our results; no such polymorphisms have yet been correlated strongly with drugresistant epilepsy. In addition, overexpression of $\mathrm{ABCB} 1$ by parenchymal glial cells in the brain, which effectively constitutes a second blood-brain barrier, has been identified in resected specimens from subjects with drug-resistant epilepsy and is confined to the epileptogenic region. ${ }^{9}$ The majority (80 percent) of such surgically treated subjects become seizure-free after lesional resection, even with reduced antiepileptic-drug dosage, suggesting that localized overexpression within the epileptogenic region of the brain is more important than expression elsewhere, such as the duodenum. In mice, systemic pharmacologic inhibition of ABCB1 leads to increased levels of the ABCB1 substrate nelfinavir in brain, with only small changes in plasma levels. ${ }^{31}$

Our data show that the drug-resistant phenotype in epilepsy is associated with the ABCB1 3435 polymorphism. Although the patients with drugresistant epilepsy and those with drug-responsive epilepsy were not stratified, all were seen at one referral center and thus were not random patients. Therefore, we cannot infer from these data the probability of resistance related to $A B C B 1$ genotype in the general population of patients with epilepsy. Within the patient base studied, however, the probability of resistance is 75.3 percent for the CC genotype and 53.4 percent for the TT genotype. Therefore, genetic factors other than $A B C B 1$ or environmental factors (or both) must also contribute to the response. Probably not all antiepileptic drugs are ABCB1 substrates, since some, such as vigabatrin, are not lipophilic planar molecules. Indeed, drug resistance in epilepsy is most likely multifactorial, compounded by unidentified mechanisms.

Our results suggest new avenues for early molecular prediction of drug resistance in patients with epilepsy. Use of antiepileptic drugs that are not $A B C B 1$ substrates, inhibition of $A B C B 1,32$ or the development of drugs that can evade $A B C B 1$ might improve the efficacy of treatment in some patients with drug-resistant epilepsy.

Supported by a program grant from the Medical Research Council; a grant from the Brain Research Trust, United Kingdom; a grant from the German Federal Ministry of Education and Science (01GG9846); and a Royal Society-Wolfson Research Merit Award (to Dr. Goldstein).

Dr. Weale reports holding equity interests in Genostics.

We are indebted to the participating patients and physicians and to Drs. P. Abou-Sleiman, P. Dixon, M. Sweeney, N. Khan, and J. Wilson.
REFERENCES

1. Casetta I, Granieri E, Monetti VC, et al. Early predictors of intractability in childhood epilepsy: a community-based case-control study in Copparo, Italy. Acta Neurol Scand 1999;99:329-33.

2. Gelisse P, Genton P, Thomas P, Rey M,
Samuelian JC, Dravet C. Clinical factors of drug resistance in juvenile myoclonic epilepsy. J Neurol Neurosurg Psychiatry 2001; 70:240-3.

3. MacDonald BK, Johnson AL, Goodridge DM, Cockerell OC, Sander JW, Shorvon SD.
Factors predicting prognosis of epilepsy after presentation with seizures. Ann Neurol 2000;48:833-41. [Errata, Ann Neurol 2001; 49:547, 50:830.]

4. Ling V. Multidrug resistance: molecular mechanisms and clinical relevance. Can- 
cer Chemother Pharmacol 1997;40:Suppl: S3-S8.

5. Loscher W, Potschka H. Role of multidrug transporters in pharmacoresistance to antiepileptic drugs. J Pharmacol Exp Ther 2002;301:7-14.

6. Tishler DM, Weinberg KI, Hinton DR, Barbaro N, Annett GM, Raffel C. MDR1 gene expression in brain of patients with medically intractable epilepsy. Epilepsia 1995;36: 1-6.

7. Sisodiya SM, Heffernan J, Squier MV. Over-expression of P-glycoprotein in malformations of cortical development. Neuroreport 1999;10:3437-41.

8. Sisodiya SM, Lin W-R, Squier MV, Thom M. Multidrug-resistance protein 1 in foca cortical dysplasia. Lancet 2001;357:42-3.

9. Sisodiya SM, Lin WR, Harding BN, Squier MV, Thom M. Drug resistance in epilepsy: expression of drug resistance proteins in common causes of refractory epilepsy. Brain 2002;125:22-31.

10. Lazarowski A, Sevlever G, Taratuto A, Massaro M, Rabinowicz A. Tuberous sclerosis associated with MDR1 gene expression and drug-resistant epilepsy. Pediatr Neuro 1999;21:731-4.

11. Dombrowski SM, Desai SY, Marroni M et al. Overexpression of multiple drug resistance genes in endothelial cells from patient with refractory epilepsy. Epilepsia 2001;42: 1501-6.

12. Hoffmeyer S, Burk O, von Richter O, et al. Functional polymorphisms of the human multidrug-resistance gene: multiple sequence variations and correlation of one allele with P-glycoprotein expression and activity in vivo. Proc Natl Acad Sci U S A 2000;97:3473-8.

13. Roy-Engel AM, Carroll ML, Vogel E, et al. Alu insertion polymorphisms for the study of human genomic diversity. Genetics 2001;159:279-90
14. Carroll ML, Roy-Engel AM, Nguyen SV, et al. Large-scale analysis of the Alu Ya5 and Yb8 subfamilies and their contribution to human genomic diversity. J Mol Biol 2001; 311:17-40.

15. Cascorbi I, GerloffT, Johne A, et al. Frequency of single nucleotide polymorphisms in the P-glycoprotein drug transporter MDR1 gene in white subjects. Clin Pharmacol Ther 2001;69:169-74.

16. Tanabe M, Ieiri I, Nagata N, et al. Expression of P-glycoprotein in human placenta: relation to genetic polymorphism of the multidrug resistance (MDR)-1 gene. J Pharmacol Exp Ther 2001;297:1137-43.

17. Reich DE, Goldstein DB. Detecting association in a case-control study while correcting for population stratification. Genet Epidemiol 2001;20:4-16.

18. Weir BS. Genetic-data analysis II: methods for discrete population genetic data. Sunderland, Mass.: Sinauer, 1996.

19. Gottesman MM, Fojo T, Bates SE. Multidrug resistance in cancer: role of ATPdependent transporters. Nat Rev Cance 2002;2:48-58.

20. Llorente L, Richaud-Patin Y, Diaz-Borjon A, et al. Multidrug resistance-1 (MDR-1) in rheumatic autoimmune disorders. I. Increased P-glycoprotein activity in lymphocytes from rheumatoid arthritis patients might influence disease outcome. Joint Bone Spine 2000;67:30-9.

21. Farrell RJ, Murphy A, Long A, et al. High multidrug resistance (P-glycoprotein 170) expression in inflammatory bowel disease patients who fail medical therapy. Gastroenterology 2000;118:279-88.

22. Hitzl M, Drescher S, van der Kuip H, et l. The C3435T mutation in the human MDR1 gene is associated with altered efflux of the P-glycoprotein substrate rhodamine 23 from CD56+ natural killer cells. Pharmacogenetics 2001;11:293-8.
23. Fellay J, Marzolini C, Meaden ER, et al. Response to antiretroviral treatment in HIV-1-infected individuals with allelic variants of the multidrug resistance transporter 1: a pharmacogenetics study. Lancet 2002; 359:30-6.

24. Drescher S, Schaeffeler E, Hitzl M, et al. MDR1 gene polymorphisms and disposition of the P-glycoprotein substrate fexofenadine. Br J Clin Pharmacol 2002;53:526-34.

25. Kim RB, Leake BF, Choo EF, et al. Identification of functionally variant MDR1 alleles among European Americans and African Americans. Clin Pharmacol Ther 2001; 70:189-99.

26. Goldstein DB. Islands of linkage disequilibrium. Nat Genet 2001;29:109-11.

27. Daly MJ, Rioux JD, Schaffner SF, Hudson TJ, Lander ES. High-resolution haplotype structure in the human genome. Nat Genet 2001;29:229-32.

28. Shapiro AB, Ling V. The mechanism of ATP-dependent multidrug transport by P-glycoprotein. Acta Physiol Scand Suppl 1998;643:227-34

29. Schinkel AH. P-glycoprotein, a gatekeeper in the blood-brain barrier. Adv Drug Deliv Rev 1999;36:179-94

30. Kerb R, Aynacioglu AS, Brockmoller J, et al. The predictive value of MDR1, CYP2C9, and CYP2C19 polymorphisms for phenytoin plasma levels. Pharmacogenomics J 2001; 1:204-10.

31. Choo EF, Leake B, Wandel C, et al. Pharmacological inhibition of P-glycoprotein transport enhances the distribution of HIV-1 protease inhibitors into brain and testes. Drug Metab Dispos 2000;28:655-60.

32. Tai HL. Technology evaluation: valspodar, Novartis AG. Curr Opin Mol Ther 2000; 2:459-67.

Copyright (c) 2003 Massachusetts Medical Society.

POSTING PRESENTATIONS AT MEDICAL MEETINGS ON THE INTERNET

Posting an audio recording of an oral presentation at a medical meeting on the Internet, with selected slides from the presentation, will not be considered prior publication. This will allow students and physicians who are unable to attend the meeting to hear the presentation and view the slides. If there are any questions about this policy, authors should feel free to call the Journal's Editorial Offices. 